\title{
2D Shape Deformation Using Nonlinear Least Squares Optimization
}

\author{
Paper ID: 20
}

\begin{abstract}
This paper presents a novel 2D shape deformation algorithm based on nonlinear least squares optimization. The algorithm aims to preserve two local shape properties: Laplacian coordinates of the boundary curve and local area of the shape interior, which are together represented in a non-quadric energy function. An iterative Gauss-Newton method is used to minimize this nonlinear energy function. The result is an interactive shape deformation system that can achieve physically plausible results that are hard to achieve with previous linear least squares methods. Besides preserving local shape properties, we also introduce a scheme to preserve the global area of the shape which is useful for deforming incompressible objects.
\end{abstract}

\section{Introduction}

2D shape deformation is a useful tool in various applications such as realtime live performance and enriching graphical user interfaces. A good shape deformation system aims to produce visually pleasing results with simple operations and provide interactive feedback to users. Many techniques have been proposed to achieve a balance between these two objectives.

Free form deformation (FFD) [15] and skeleton-based methods [8] are widely used methods in commercial softwares nowadays. They run fast, however, setting FFD domains and skeleton configuration is tedious and it is laborious to manipulate many control points in FFD. Physicallybased simulations [4] can achieve pleasing results but with very low convergence.

Recently, Igarashi et al. [6] presents an interactive system that allows the user to deform a $2 \mathrm{D}$ shape by manipulating a few points. The shape is represented by a triangle mesh and the user moves several vertices of the mesh as constrained handles. The system then computes the positions of the remaining free vertices by minimizing the distortion of each triangle. To make the problem linear, they present a two-step solution: the first step to compute the rotation and the second step to compute the scale. As the
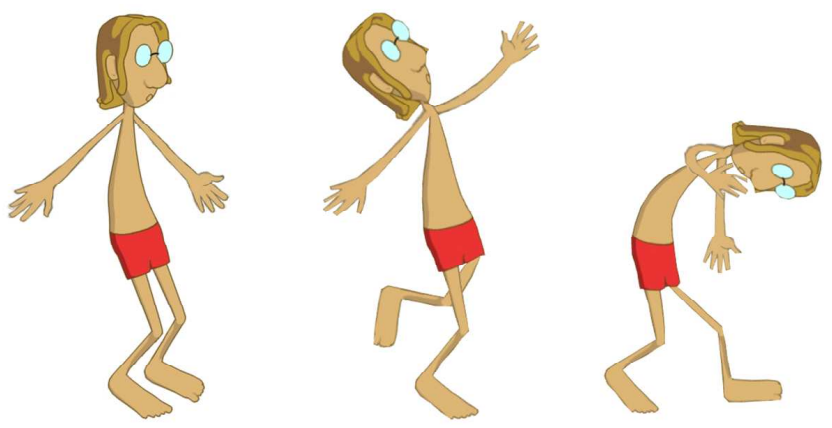

Figure 1. 2D Deformation of Cartoon man. Left:the original shape; Middle and right: the deformation results generated by our algorithm.

authors admitted, the two-step algorithm is merely a practical approximation to achieve interactive performance and may cause unplausible results in some cases due to its linear nature.

In this paper we present a novel 2D shape deformation algorithm based on nonlinear least squares optimization. The algorithm aims to preserve two geometric properties of 2D shapes: the Laplacian coordinates of the boundary curve of the shape and local areas inside the shape, which are together represented in a non-quadric energy function. Instead of linearizing these nonlinear properties, we cast the problem as an nonlinear least squares minimization and solve it using an iterative method. The resulting system is able to achieve physically plausible deformation results and runs interactively. Besides preserving local shape properties, we also introduce a scheme to preserve the global area of the shape which is useful for deforming incompressible objects.

\subsection{Related Work}

There are a lot of literatures on shape deformation. Here we only overview those most related works to 2D shape deformation.

The best known method for shape deformation may be 
free form deformation (FFD) [10, 13, 15]. In FFD, a shape is embedded in a lattice, then is deformed by moving the control points of the lattice. While FFD is simple and easy to use, it does not take into account the natural way in which shapes features are controlled. For example, many animals have a skeleton. Skeleton-based deformation [8] provides an intuitive approach to control deformation of animal-like shapes. Skeleton-based algorithms define the position of a point as a weighted linear combination of the initial state of the point projected into several moving coordinate frames, corresponding to the bones, which is usually specified manually. Appropriate weight selection is a painful process.

To achieve physically plausible deformation, physicallybased simulations can be employed [2, 4, 7]. Among these methods, the most popular one is the mass-spring models [4]. However, it is too slow to converge and needs careful tuning of various parameters. Finite-element methods [2] can get more physically accurate simulation at the expense of long solving time. Therefore, they are inappropriate for interactive deformation applications. The ArtDefo system [7] can run interactively, but is limited to small deformations.

Gradient domain techniques [6, 9, 17, 18, 19] cast deformation as an energy minimization problem. The energy function contains both the term for detail-preserving constraint and the term for position constraint. The detailpreserving constraint is nonlinear because it involves both the differentials for local details and the local transformations which are position dependent. For computational efficiency, existing techniques convert this nonlinear constraint into a linear one by using various approximations including local linearization of transformation [17], transformation interpolation from handles $[9,18,19]$ and the decomposition of rotation and scaling computation [6]. The price for employing these least squares minimization schemes is suboptimal deformation results.

Our algorithm can be viewed as a variant of recent nonlinear mesh deformation methods $[1,5,16]$. All these methods try to minimize a nonlinear energy function representing some kinds of local properties of the surface. Instead of $3 \mathrm{D}$ mesh, our algorithm only deal with $2 \mathrm{D}$ shapes. Therefore the local properties we are trying to preserve are quite different from those in 3D surface.

\section{Overview}

The input of our algorithm is a $2 \mathrm{D}$ shape (see Figure 2(a)), with the boundary represented as a simple closed polygon. The algorithm automatically inserts a set of points into the interior region of the shape and generates a graph by connecting the vertices of the boundary polygon and the inside points (see Figure 2(b)). Then the user can drag the points to deform the shape.
The algorithm aims to preserving two local properties: Laplacian coordinates of the boundary curve and the local area inside the shape. The Laplacian coordinates represent the local details of the shape boundary and are widely used in 3D mesh deformation methods $[9,17,19]$. While preserving Laplacian coordinates often produces good deformation results $3 \mathrm{D}$ meshes, it is not enough to produce visually pleasing deformation results for $2 \mathrm{D}$ shapes (see Figure 3 ). Therefore, we also try to preserve the local areas inside the shape. To achieve this goal, we build a graph and introduce two new local properties for the graph: the relative position (mean value coordinates) of each interior point with respect to its neighbors and the length of each edge. To control a deformation, the user inputs the deformed positions for a subset of the graph points. The deformed positions of all graph points are then obtained by minimizing an energy function that consists of four parts: Laplacian coordinate preserving, mean value coordinates preserving, edge length preserving and position constraints (see details in Section $3)$.
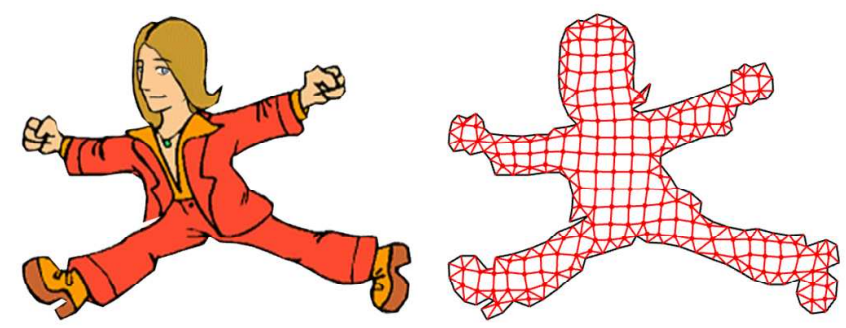

Figure 2. 2D shape and its interior graph.

To build the interior graph, one can generate a triangulated mesh inside the boundary polygon like [6]. We instead adopt an easier approach similar to the volumetric graph construction in [19]. It consists of four steps (see Figure 6 in [19]). Firstly, construct an inner polygon for the boundary polygon by offsetting each vertex a distance in the direction opposite its normal; Secondly, embed the two polygons in a lattice, remove lattice nodes outside the inner polygon; Thirdly, build edge connections among the two polygons and lattice nodes; Finally, simplify the graph using edge collapse and smooth the graph.

Now we have a 2D graph $(V, E)$, where $V$ is the set of $n$ vertices in the graph, and $E$ is the set of edges. $V$ includes two subsets: $V_{p}$, which contains $m$ vertices of polygon, and $V_{g}$, which contains $(n-m)$ interior points. Similarly, The edge set $E$ can be divided into two sets: $E_{p}$, which contains polygon edges, and $E_{g}$ which represents the rest edges in graph.

The remainder of this paper is organized as follows. The following section explains the three local properties in detail. In Section 4, we combine all the local properties to- 
gether and present an iterative solver to compute the deformation results efficiently. Section 5 describes how to preserve the global area in our algorithm, which is useful for deforming incompressible objects. Experimental results are shown in Section 6, and the paper concludes with some discussion of future work in Section 7.

\section{Local Properties Preserving}

This section describes the three local properties: Laplacian coordinates, mean-valued coordinates and edge length. Laplacian coordinates represents the local details of the boundary polygon. Mean-valued coordinates and edge length are used to achieve local area preserving.

\subsection{Curve Laplacian Coordinates}

Curve Laplacian is defined for each point in $V_{p}$ and it is analogous to the Laplacian on 3D meshes. Specifically, the curve Laplacian coordinate $\delta_{i}$ of point $v_{i}$ is computed as the difference between $v_{i}$ and the average of its neighbors on the curve:

$$
\delta_{i}=\mathscr{L}_{p}\left(v_{i}\right)=v_{i}-\left(v_{i-1}+v_{i+1}\right) / 2,
$$

where $v_{i-1}$ and $v_{i+1}$ are the adjacent points to $v_{i}$ on the curve; $\mathscr{L}_{p}$ is called the Laplace operator of the curve.

To preserve the Laplacian coordinates during deformation, we try to minimize the following energy function:

$$
\sum_{v_{i} \in V_{p}}\left\|\mathscr{L}_{p}\left(v_{i}\right)-\delta_{i}\right\|^{2}
$$

which is equivalent to the matrix form:

$$
\left\|\mathbf{L}_{p} \mathbf{V}_{p}-\delta\left(\mathbf{V}_{p}\right)\right\|^{2}
$$

where $\mathbf{V}_{p}$ is the point positions of the boundary polygon and $\mathbf{L}_{p}$ is a $m \times m$ matrix, called Laplace matrix; $\delta$ is the vector of Laplacian coordinates. Note that we view $\delta$ as a general function of the point positions $\mathbf{V}_{p}$ instead of a linear function of $\mathbf{V}_{p}$ like [17].

To make the description clear in the following, we expand $\mathbf{L}_{p}$ to a $m \times n$ matrix $\mathbf{L}$ by adding zero elements. Then Equation (1) can be rewritten as:

$$
\|\mathbf{L V}-\delta(\mathbf{V})\|^{2}
$$

\subsection{Mean Value Coordinates}

For each point $v_{i}$ in $V_{g}$, we want to maintain its relative position with respect to its neighboring points during deformation. To do this, we first compute its mean value coordinates [3] in the polygon formed by its neighboring points:

$$
w_{i, j}=\frac{\tan \left(\alpha_{j} / 2\right)+\tan \left(\alpha_{j+1} / 2\right)}{\left|v_{i}-v_{j}\right|},
$$

where $\alpha_{j}$ is the angle formed by the vector $v_{j}-v_{i}$ and $v_{j+1}-v_{i}$. Normalizing each weight function $w_{i, j}$ by the sum of all weight functions yields the mean value coordinates of $v_{i}$ with respect to its neighboring points.

According to the the property of the mean value coordinate, We have:

$$
v_{i}-\sum_{(i, j) \in E} w_{i, j} * v_{j}=0, \text { for } v_{i} \in V_{g},
$$

which can also be represented as a matrix form:

$$
\mathbf{M}_{g} \mathbf{V}_{g}=0
$$

where $\mathbf{M}_{g}$ is a $(n-m) \times(n-m)$ matrix. Similar to $\mathbf{L}_{p}, \mathbf{M}_{g}$ can be expanded to a $(n-m) \times n$ matrix $\mathbf{M}$ by adding zero elements.

To preserve the mean value coordinates during deformation, we minimize the following energy function:

$$
\|\mathbf{M V}\|^{2}
$$

\subsection{Edge Lengths}

Note that mean value coordinates are invariant to scaling. Preserving mean value coordinates is not enough to preserve the local areas inside the shape. Therefore, we further try to preserve edge length during deformation.

We penalize the edge length changes for all edges in $E_{g}$ using the following energy:

$$
\sum_{(i, j) \in E_{g}}\left\|\left(v_{i}-v_{j}\right)-e\left(v_{i}, v_{j}\right)\right\|^{2},
$$

where $e\left(v_{i}, v_{j}\right)=\frac{\widetilde{i_{i, j}}}{l_{i, j}}\left(v_{i}-v_{j}\right) ; l_{i, j}$ is the current length of edge $(i, j)$ and $\widetilde{l_{i, j}}$ is the rest length before deformation.

Note that the energy associated with each edge is computed in vector form such that the whole energy in Equation (4) can be represented in a matrix form:

$$
\|\mathbf{H V}-e(\mathbf{V})\|^{2}
$$

where $\mathbf{H}$ is a $\left|E_{g}\right| \times n$ matrix.

\section{Shape Deformation Using Nonlinear Least Squares Optimization}

\subsection{Deformation Energy}

To control a deformation, the user inputs the deformed positions for a subset $S$ of the graph points. This information is used to compute the deformed positions of all graph 

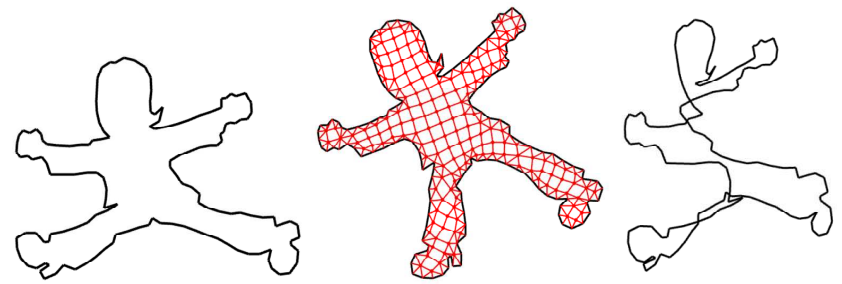

Figure 3. Deformation results with and without local area preservation. Left: original shape; Middle: deformation result which preserves Laplacian coordinates only; Right: deformation result which preserves both Laplacian coordinates and local area.

points by minimizing the following energy which sums up all energy terms:

$$
\|\mathbf{L V}-\boldsymbol{\delta}(\mathbf{V})\|^{2}+\|\mathbf{M V}\|^{2}+\|\mathbf{H V}-e(\mathbf{V})\|^{2}+\|\mathbf{C V}-\mathbf{U}\|^{2},
$$

where $\|\mathbf{C V}-\mathbf{U}\|^{2}$ represents the position constraints specified by the user; $\mathbf{C}$ is a $|S| \times n$ matrix and $\mathbf{U}$ is a vector of dimension $|S|$ representing the target positions specified by the user.

The above energy minimization problem can be reformulated as the following:

$$
\min _{\mathbf{V}}\|\mathbf{A V}-\mathbf{b}(\mathbf{V})\|^{2}
$$

where:

$$
\mathbf{A}=\left(\begin{array}{c}
\mathbf{L} \\
\mathbf{M} \\
\mathbf{H} \\
\mathbf{C}
\end{array}\right), \mathbf{b}(\mathbf{V})=\left(\begin{array}{c}
\delta(\mathbf{V}) \\
0 \\
e(\mathbf{V}) \\
\mathbf{U}
\end{array}\right)
$$

Note that the matrix $\mathbf{A}$ is dependent only on the graph before deformation while $\mathbf{b}$ is dependent on the current point positions $\mathbf{V}$. This is a nonlinear least squares problem. Previous methods try to make this a linear least squares problem solvable either by removing the dependency of $\mathbf{b}$ on $\mathbf{V}$ or by using a linear approximation for $\mathbf{b}$. In the following, we introduce an iterative Gauss-Newton method [11] to solve this nonlinear problem directly.

\subsection{Nonlinear Least Squares Optimization}

The iterative Gauss-Newton method solve the problem in the following way:

$$
\min _{\mathbf{V}^{k+1}}\left\|\mathbf{A} \mathbf{V}^{k+1}-\mathbf{b}\left(\mathbf{V}^{k}\right)\right\|^{2}
$$

where $\mathbf{V}^{k}$ is the point positions solved from the $k$-th iteration and $\mathbf{V}^{k+1}$ is the point positions we want to solve at iteration

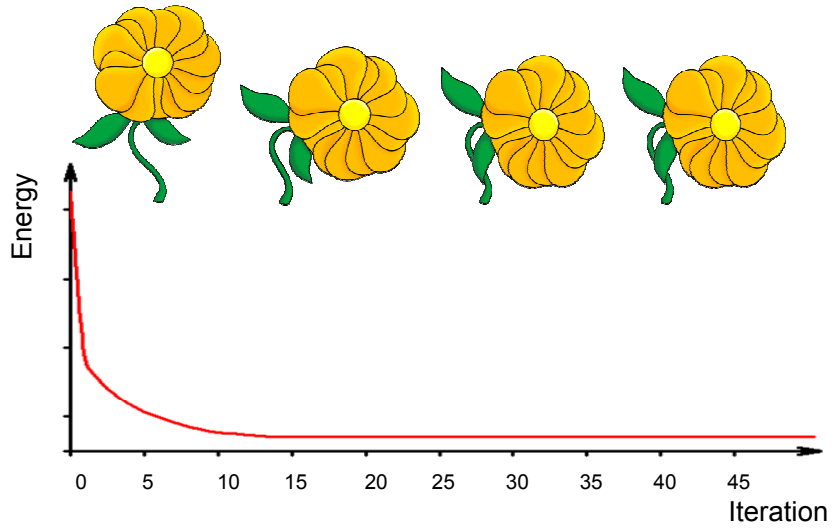

Figure 4. Convergence of our iterative solver. The red curve indicates energy.

$k+1$. Since $\mathbf{b}\left(\mathbf{V}^{k}\right)$ is known at the current iteration, Equation (8) can be solved through a linear least squares system:

$$
\mathbf{V}^{k+1}=\left(\mathbf{A}^{T} \mathbf{A}\right)^{-1} \mathbf{A}^{T} \mathbf{b}\left(\mathbf{V}^{k}\right)=\mathbf{G b}\left(\mathbf{V}^{k}\right) .
$$

Let $\mathbf{G}=\left(\mathbf{A}^{T} \mathbf{A}\right)^{-1} \mathbf{A}^{T}$. Since $\mathbf{A}$ is dependent only on the graph before deformation, $\mathbf{G}$ can be precomputed before deformation and is fixed during deformation. Therefore, only a back substitution is executed for each iteration. In this way, the deformation algorithm is able to run interactively.

During each iteration, $\mathbf{b}$ is computed according to the point positions $\mathbf{V}^{k}$ from last iteration. In other words, we need to compute $\delta\left(\mathbf{V}^{k}\right)$ and $e\left(\mathbf{V}^{k}\right)$.

$e\left(\mathbf{V}^{k}\right)$ is computed as follows:

$$
e\left(v_{i}^{k}, v_{j}^{k}\right)=\frac{\widetilde{l_{i, j}}}{\left|v_{i}^{k}-v_{j}^{k}\right|}\left(v_{i}^{k}-v_{j}^{k}\right), \text { for }(i, j) \in E_{g} .
$$

Computing the new Laplacian coordinates $\delta\left(\mathbf{V}^{k}\right)$ is a little bit complicated. Specifically, we compute a transform matrix $T_{i}^{k}$ for each point $v_{i} \in V_{p}$ :

$$
\delta\left(v_{i}^{k}\right)=T_{i}^{k} \boldsymbol{\delta}\left(v_{i}^{0}\right),
$$

where $\delta\left(v_{i}^{0}\right)$ is the curve Laplacian coordinate before deformation.

By taking $v_{i}^{0}$ and $v_{i}^{k}$ as the rotation centers, the transform matrix $T_{i}^{k}$ can be computed by minimizing the following energy [14]:

$$
\sum_{(i, j) \in E_{p}}\left\|T_{i}^{k}\left(v_{j}^{0}-v_{i}^{0}\right)-\left(v_{j}^{k}-v_{i}^{k}\right)\right\|^{2}
$$

Taking the derivatives to all coefficients of $T_{i}^{k}$ to be zero, we can get:

$T_{i}^{k}=\sum_{(i, j) \in E_{p}}\left(v_{j}^{k}-v_{i}^{k}\right)\left(v_{j}^{0}-v_{i}^{0}\right)^{T}\left(\sum_{(i, j) \in E_{p}}\left(v_{j}^{0}-v_{i}^{0}\right)\left(v_{j}^{0}-v_{i}^{0}\right)^{T}\right)^{-1}$ 
Note that $\left(\sum_{(i, j) \in E_{p}}\left(v_{j}^{0}-v_{i}^{0}\right)\left(v_{j}^{0}-v_{i}^{0}\right)^{T}\right)^{-1}$ depends on the original shape only. It can also be precomputed to accelerate the algorithm.

\section{Global Area Preserving}

In this section, we introduce how to preserve the global area of the shape to simulate incompressible 2D object. As you will see in the following, global area preserving is handled as a hard constraint of the nonlinear least squares problem (Equation (7)) and the iterative solver described above can be adapted to solve this constrained problem efficiently.

The area of a polygon is computed using the coordinates of the polygon points: $g\left(\mathbf{V}_{p}\right)=\frac{1}{2} \sum_{i=0}^{m}\left(x_{i} y_{i+1}-x_{i+1} y_{i}\right)$, where $\left(x_{i}, y_{i}\right)$ is the coordinate of point $v_{i}$. Then the global area constraint can be formulated as following:

$$
g(\mathbf{V})-\widetilde{g}=0
$$

where $\widetilde{g}$ is the area of the original shape before deformation.

Since the global area constraint is a nonlinear function of the coordinates of the polygon points, it can not be written into a matrix form. Thus we treat this constraint as a hard constraint and extend Equation (7) to:

$$
\min _{\mathbf{V}}\|\mathbf{A V}-\mathbf{b}(\mathbf{V})\|^{2} \text {, subject to } g(\mathbf{V})-\widetilde{g}=0
$$

This constrained non-linear least squares problem can also be solved by extending the iterative solver (Equation (8)) to the following formula:

$$
\min _{\mathbf{V}^{k+1}}\left\|\mathbf{A} \mathbf{V}^{k+1}-\mathbf{b}\left(\mathbf{V}^{k}\right)\right\|^{2} \text {, subject to } g\left(\mathbf{V}^{k+1}\right)-\widetilde{g}=0
$$

Let

$$
\mathbf{h}=\mathbf{V}^{k+1}-\mathbf{V}^{k}
$$

$\mathbf{A} \mathbf{V}^{k+1}-\mathbf{b}\left(\mathbf{V}^{k}\right)$ can be reformulated as a new function $l(\mathbf{h})$ which only depends on $\mathbf{h}$ :

$$
\begin{aligned}
l(\mathbf{h}) & =\mathbf{A} \mathbf{V}^{k+1}-\mathbf{b}\left(\mathbf{V}^{k}\right) \\
& =\mathbf{A}\left(\mathbf{V}^{k}+\mathbf{h}\right)-\mathbf{b}\left(\mathbf{V}^{k}\right) \\
& =\mathbf{A h}+\mathbf{A} \mathbf{V}^{k}-\mathbf{b}\left(\mathbf{V}^{k}\right) .
\end{aligned}
$$

The problem (11) is converted to:

$$
\min _{\mathbf{h}} \frac{1}{2}\|l(\mathbf{h})\|^{2}, \text { subject to } g\left(\mathbf{V}^{k}+\mathbf{h}\right)-\widetilde{g}=0
$$

By locally linearizing

$$
g\left(\mathbf{V}^{k}+\mathbf{h}\right) \approx g\left(\mathbf{V}^{k}\right)+\mathbf{J}_{g}\left(\mathbf{V}^{k}\right) \mathbf{h},
$$

and applying Lagrange multipliers [12] with Newton's method, the solution to (13) is:

$$
\begin{aligned}
& \mathbf{h}=-\left(\mathbf{A}^{T} \mathbf{A}\right)^{-1}\left(\mathbf{A}^{T} \mathbf{S}+\mathbf{J}_{g}^{T} \lambda\right) \\
& \lambda=-\left(\mathbf{J}_{g}\left(\mathbf{A}^{T} \mathbf{A}\right)^{-1} \mathbf{J}_{g}^{T}\right)^{-1}\left(t-\mathbf{J}_{g}\left(\mathbf{A}^{T} \mathbf{A}\right)^{-1} \mathbf{A}^{T} \mathbf{S}\right)
\end{aligned}
$$

where $\mathbf{J}_{g}$ is the Jacobian of $g, \mathbf{S}=\mathbf{b}\left(\mathbf{V}^{k}\right)-\mathbf{A} \mathbf{V}^{k}$, and $t=$ $\widetilde{g}-g\left(\mathbf{V}^{k}\right)$.

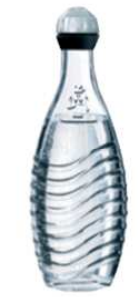

area $=1.0$

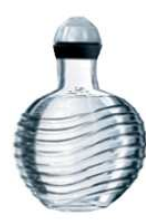

area $=1.0$

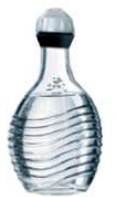

area $=0.6996$

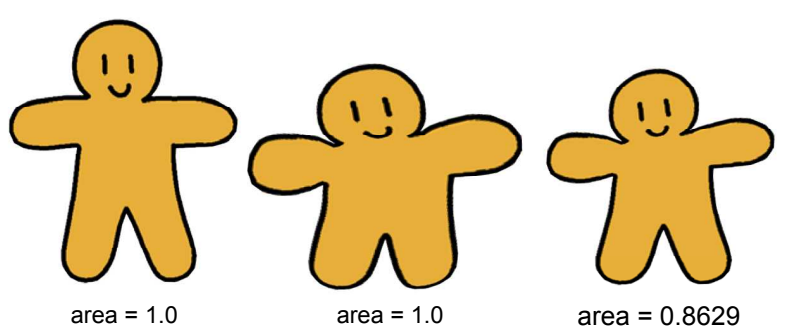

Figure 5. Deformation with (middle) global area constraint and without (right) global area constraint. The original 2D shapes are on the left.

\section{Experimental Results}

We have implemented the described deformation algorithm on a $3.2 \mathrm{GHz}$ Pentium 4 workstation with $1 \mathrm{~GB}$ memory. Table 1 shows the data statistics and timings for several models presented in this paper. The solving time means the per-iteration cost. The number of the iterations need for convergence of the solver varies significantly depending on many factors such as the shape itself and the magnitude of the deformation. For models used in this paper, the average number is 10 . Therefore, the performance of our deformation system is comparable to previous linear methods [6]. As shown in the accompanying video, our system runs in real-time.

In Figure 4, we show an example to demonstrate the convergence of our iterative solver. The curve is generated by setting the constraint points to the target position and let the solver iterate until convergence. In this example, the solver converges after about 10 iterations. Consider the solving time of our solver(see Table 1), it is very fast.

Figure 3 compares the deformation results with and without local area preserving. If we only preserve Laplacian coordinate, the deformation result looks unnatural with obvious self-intersection. By adding graph mean-value coordinates and edge length constraints to control the local area inside the $2 \mathrm{D}$ shape, the result looks much more pleasing.

For most examples presented in this paper, our results are as good as those results generated by the linear method [6]. 
In some case, our nonlinear least squares optimization leads to more physically plausible result than [6] does. Figure 6 shows the deforming results of a stick.

We have tested our deformation algorithm on various kinds of 2D shapes. Figure 7 shows the deformation of a flower. the stem of the flower is deformed naturally, and the shape of the flower is preserved well. Our system can also be used to deform cartoon character (Figure 8). Figure 8 shows that large scale deformation of the legs of the cartoon man. Figure 9 illustrates the deformation result of a horse. The details at the tail and back of the horse are well preserved even in large deformation.

Figure 5 demonstrates the effect of global area constraint. The rest shape is compressed to show the effect. The deformation result with the global area constraint is fatter than the result without global area constraint. This is useful to simulate incompressible objects.

\begin{tabular}{|c|c|c|c|}
\hline 2D Shape & flower & horse & man \\
\hline \# Boundary Vertices & 114 & 247 & 143 \\
\hline \# Interior Vertices & 256 & 189 & 163 \\
\hline Precomputing time & $22 \mathrm{~ms}$ & $22.7 \mathrm{~ms}$ & $18.3 \mathrm{~ms}$ \\
\hline Solving time & $0.589 \mathrm{~ms}$ & $0.593 \mathrm{~ms}$ & $0.470 \mathrm{~ms}$ \\
\hline
\end{tabular}

Table 1. Statistics and timing.

\section{Conclusion and Future Work}

We have described a real-time 2D shape deformation algorithm based on nonlinear least squares optimization. Our algorithm is able to preserve both local and global properties of the input shape. The nonlinear nature of our algorithm makes it outperforms previous linear methods.

For future work, we are planning to add more useful constraints, such as skeleton constraint, into the deformation energy. Our algorithm can also be applied to 2D cartoon animation retargeting by defining a set of corresponding points between $2 \mathrm{D}$ shapes.

\section{References}

[1] O. K.-C. Au, C.-L. Tai, L. Liu, and H. Fu. Mesh editing with curvature flow laplacian operator. Technical report, Computer Science Technical Report, HKUST-CS05-10, 2005.

[2] G. Celniker and D. Gossard. Deformable curve and surface finite-elements for free-form shape design. In SIGGRAPH 91 Conference Proceedings, pages 257-266, 1991.

[3] M. S. Floater. Mean value coordinates. Comp. Aided Geom. Design, 20(1):19-27, 2003.

[4] S. F. F. Gibson and B. Mirtich. A Survey of Deformable Modeling in Computer Graphics. Technical report TR-9719, Mitsubishi Electric Research Laboratories, 1997.
[5] J. Huang, X. Shi, X. Liu, K. Zhou, L. Wei, S. Teng, H. Bao, B. Guo, and H.-Y. Shum. Subspace gradient domain deformation. In SIGGRAPH 2006 Conference Proceedings, to appear.

[6] T. Igarashi, T. Moscovich, and J. F. Hughes. As-rigidas-possible shape manipulation. ACM Trans. Graphics, 24(3):1134-1141, 2005.

[7] D. L. James and D. K. Pai. Artdefo: Accurate real time deformable objects. In SIGGRAPH 99 Conference Proceedings, pages 65-72, 1999.

[8] J. P. Lewis, M. Cordner, and N. Fong. Pose space deformation: a unified approach to shape interpolation and skeletondriven deformation. In SIGGRAPH 2000 Conference Proceedings, pages 165-172, 2000.

[9] Y. Lipman, O. Sorkine, D. Levin, and D. Cohen-Or. Linear rotation-invariant coordinates for meshes. ACM Trans. Graphics, 24(3):479-487, 2005.

[10] R. MacCracken and K. Joy. Free-form deformations with lattices of arbitrary topology. In SIGGRAPH 96 Conference Proceedings, pages 181-188, 1996.

[11] K. Madsen, H. Nielsen, and O. Tingleff. Methods for nonlinear least squares problems. Technical report, Informatics and Mathematical Modelling, Technical University of Denmark, 2004.

[12] K. Madsen, H. Nielsen, and O. Tingleff. Optimization with constraints. Tech. rep. Informatics and Mathematical Modelling, Technical University of Denmark, 2004.

[13] T. Milliron, R. Jensen, R. Barzel, and A. Finkelstein. A framework for geometric warps and deformations. ACM Trans. Graphics, 21(1):20-51, 2002.

[14] M. Müller, B. Heidelberger, M. Teschner, and M. Gross. Meshless deformations based on shape matching. ACM Trans. Graphics, 24(3):471-478, 2005.

[15] T. Sederberg and S. Parry. Free-form deformation of solid geometric models. SIGGRAPH 86 Conference Proceedings, 20(4):151-160, 1986.

[16] A. Sheffer and V. Kraevoy. Pyramid coordinates for morphing and deformation. In Proceedings of 3DPVT, 2004.

[17] O. Sorkine, Y. Lipman, D. Cohen-Or, M. Alexa, C. Rössl, and H.-P. Seidel. Laplacian surface editing. In Symposium on Geometry Processing, pages 179-188. ACM SIGGRAPH / Eurographics, 2004.

[18] Y. Yu, K. Zhou, D. Xu, X. Shi, H. Bao, B. Guo, and H.-Y. Shum. Mesh editing with poisson-based gradient field manipulation. ACM Trans. on Graphics, 23(3):644-651, 2004.

[19] K. Zhou, J. Huang, J. Snyder, X. Liu, H. Bao, B. Guo, and H.-Y. Shum. Large mesh deformation using the volumetric graph laplacian. ACM Trans. Graphics, 24(3):496-503, 2005. 


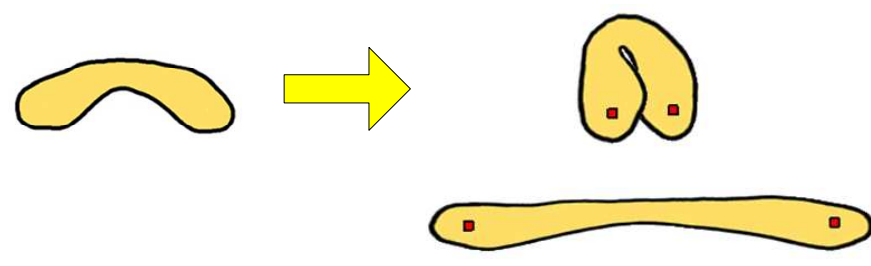

(a) rest shape

(b) our algorithm

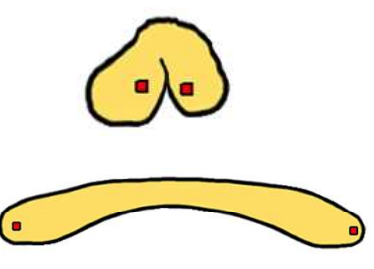

(c) [T. Igarashi et al. 2005]

Figure 6. Comparison between our algorithm and Igarashi et al.
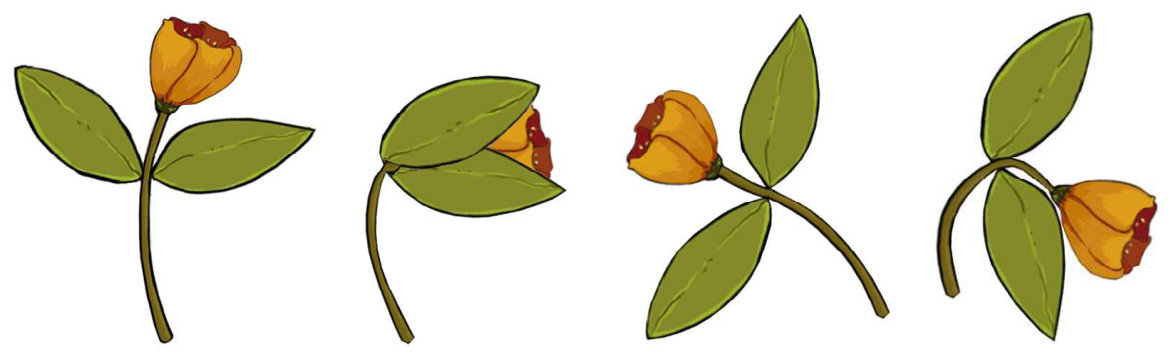

Figure 7. Deformation of a flower (left to right). Left is the rest shape.
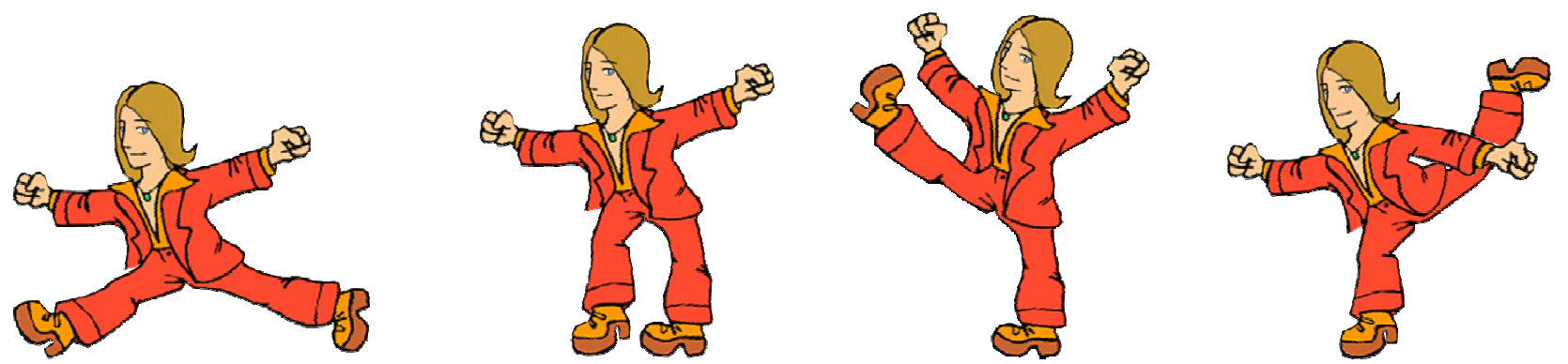

Figure 8. Deformation of a cartoon character (left to right). Left is the rest shape.
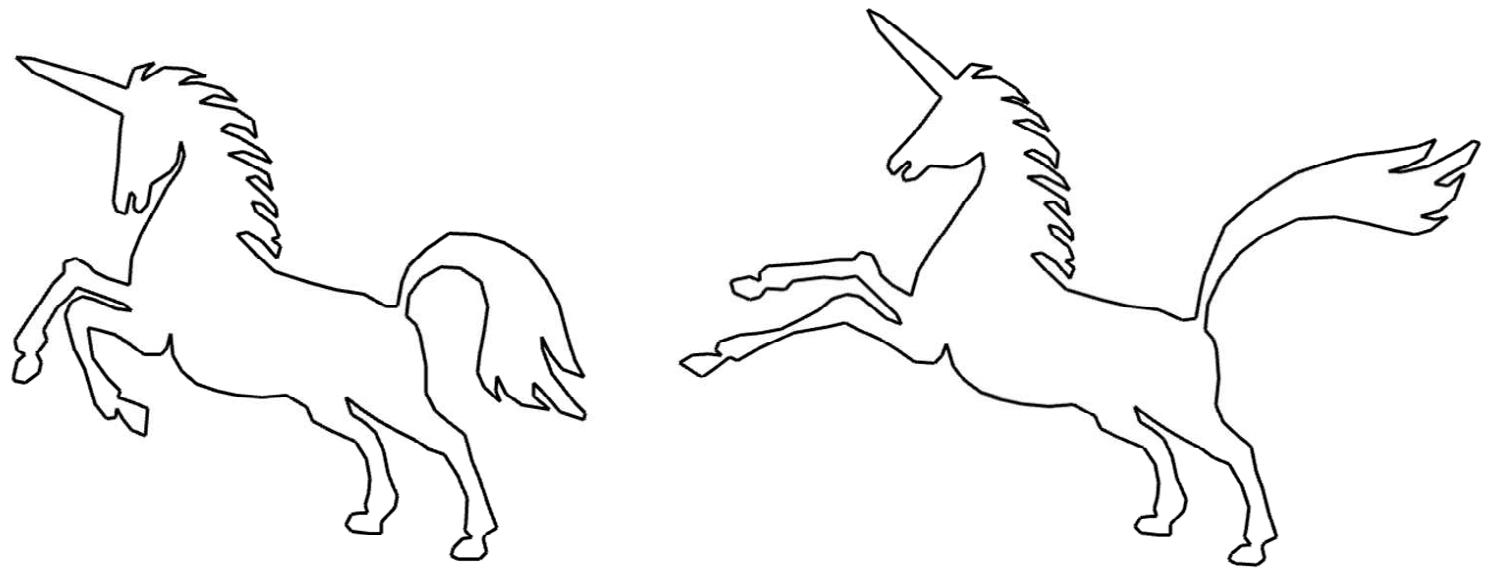

Figure 9. Deformation of a horse. Left is the rest shape. Right is the deformation result. 